\title{
Les particularités du rétinoblastome au Niger
}

\author{
Abba Kaka.H.Y \\ Service d'Ophtalmologie de l'Hôpital de National de Niamey, Niger. \\ Sylla $\boldsymbol{F}$ \\ Institut d'Ophtalmologie Tropical de l'Afrique, Bamako, Mali
}

Ali M.H

Service d'Ophtalmologie de l'Hôpital de National de Niamey, Niger.

Amza A

Service d'Ophtalmologie de l'Hôpital National de Lamordé, Niamey, Niger.

doi: 10.19044/esj.2016.v12n3p84 URL:http://dx.doi.org/10.19044/esj.2016.v12n3p84

\begin{abstract}
Aims: To describe the clinical peculiarities of retinoblastoma and to outline the difficulties in their management in our context.

Patients and Methods: We carried out a prospective study in all the cases of retinoblastoma from January 2014 to July 2015 in Niamey National Hospital. We studied: age, sex, first sign, age at first symptoms, time taken to seek medical help, anterior treatment , consanguinity, laterality, stage of tumor, extension of tumor, treatment done, survival rate.

Results: The study was about 57 kids, among which 38 boys and 19 girls giving a sex ratio of 2.The mean age at diagnosis was 32 months with extremes of 7 months and 6 years. The range 2 to 3 years were most affected with 35 cases $(61,4 \%)$. Leucocorie was the onset symptom in 50 cases (87, $7 \%)$, strabismus in 7 cases (12, 3\%). Traditional treatment was seen in 45 patients (79\%). Consanguinity was found in 45 cases (79\%), no family history of tumor was found in this study. In 38 cases (66, 66\%) diagnosis was after one year of onset, the left eye was affected in 22 patients (38, $59 \%$ ), the right eye in 21 patients $(36,84 \%)$ and it was bilateral in 14 cases (24, 56\%). All our patients were at stage $\mathrm{V}$ of Reese classification, the tumor was extra-ocular in 54 patients $(94,6 \%)$ and intra-ocular in 5, 4\% of cases. Enucleation was performed in 46 cases (80, 70\%) and 56 patients (94, 73\%) underwent chemotherapy cure. The survival rate after 18 months was of 15 , $78 \%$.

Conclusion: Late presentation, diagnosis at an advance stage, and limited treatment options are the main factors responsible for the low rate of survival in this study.
\end{abstract}


Keywords: retinoblastoma, Niger

\section{Résumé}

Objectif: Décrire les particularités cliniques du rétinoblastome et ressortir les difficultés de sa prise en charge dans notre contexte.

Patients et méthodes : Il s’agissait d'une étude prospective et descriptive de Janvier 2014 à Juillet 2015 à l’hôpital National de Niamey. Ont été inclus tout les cas de rétinoblastome dont les parents ont consenti à l'étude. Non inclus étaient les autres tumeurs de l'enfant. Les paramètres suivants ont été étudiés: l’âge, le sexe, les signes d'appel, l’âge à l'apparition des symptômes, le délai de consultation, le traitement antérieur, la notion de consanguinité, la latéralité, le stade de la maladie, l’extension de la tumeur, le traitement reçu, et le délai de survie

Résultats : la série comportait 57 cas dont 38 (66,66\%) garçons et 19 $(33,33 \%)$ filles soit un sex ratio de 2 . L’âge moyen était de 32 mois avec des extrêmes de 7 mois et 6 ans. La tranche d'ge de 2 à 3 ans était la plus représentée avec 35 cas (61,4\%).Le mode de debut était la leucocorie dans 50 cas $(87,7 \%)$ et le strabisme dans 7 cas $(12,3 \%)$. Chez 45 patients (79\%) il y avait un antécédent de traitement traditionnel. La consanguinité a été notée chez 45 cas (79\%), aucun antécédent familial de tumeur n’a été retrouvé. Chez 38 patients $(66,66 \%)$ la consultation s'est faite plus d'un an après l'apparition des symptômes. L'œil gauche était atteint dans 22 cas (38,59\%), l'œil droit dans 21 cas (36,84\%) et la tumeur était bilatérale dans 14 cas (24,56\%).Tous nos patients étaient au stade $\mathrm{V}$ de la classification de Reese. La tumeur était extra- oculaire dans 54 cas (94,6\%). Une énucléation a été faite chez 46 patients soit 80,70\% et 54 patients $(94,73 \%)$ avaient reçu une chimiothérapie. Neuf patients (15,78\%) avaient survécu au delà de 18 mois. Conclusion:Le retard à la consultation, le diagnostic à un stade avancé de la maladie et les moyens de prise en charge limités constituent les raisons fondamentales responsables du mauvais pronostic du rétinoblastome au Niger.

Mots clés: rétinoblastome, Niger

\section{Introduction}

Le rétinoblastome est une tumeur intraoculaire hautement maligne, touchant essentiellement le nourrisson et le jeune enfant; Son incidence est estimée à 1/15000 à 1/20000 naissances vivantes dans les pays industrialisés [1]. Dans ces pays le pronostic des rétinoblastomes s'est amélioré ces dernières années grâce aux progrès considérables réalisés aussi bien sur le plan du diagnostic que sur le plan thérapeutique[2]. Mais cette pathologie occasionnerait entre 3001 à 3376 décès d’enfants annuellement dont la 
plupart répartie entre l'Afrique et l'Asie. Au NIGER, on ne dispose pas de données sur cette pathologie qui menace la vue et surtout la vie du jeune enfant. Le but de cette étude est de ressortir les particularités épidémiologiques et cliniques du rétinoblastome mais aussi les difficultés liées à sa prise en charge dans notre contexte.

\section{Patients et Méthodes}

Il s'agissait d'une étude prospective et descriptive réalisée dans le service d'ophtalmologie de l'Hôpital National de Niamey de Janvier 2014 à Juillet 2015 soit une durée de 19 mois. L'étude a concerné tous les enfants qui avaient un rétinoblastome et dont les parents étaient consentants, non inclus étaient les autres tumeurs de l'. Tous les patients ont bénéficié d'un examen ophtalmologique complet dont certains sous anesthésie générale et d'autres directement au biomicroscope. Ils avaient aussi bénéficié d'un bilan biologique et radiologique et pour certains d'un scanner orbito-cérébral. Les patients ayant une masse tumorale extraorbitaire avaient bénéficiéd'une chimiothérapie néo adjuvante Les variables étudiées étaient l'âge, le sexe, les signes d'appel, l'âge à l'apparition des symptômes, le délai entre l'apparition des symptômes et la première consultation, le traitement reçu, la consanguinité, les antécédents familiaux de tumeurs oculaires, la latéralité, le stade de la maladie au moment du diagnostic, l'extension de la tumeur, traitement, et le délai de survie... le test de comparaison utilisé était celui du Chi2 avec une limite de confiance à $95 \%$.

\section{Résultats}

La série comportait 57 patients dont 38 garçons $(66,66 \%)$ et 19 filles $(33,33 \%)$ soit un sex ratio de 2 . L'âge moyen était de 32 mois avec des extrêmes de 7 mois et 6 ans. La tranche d'âge la plus atteinte était celle entre 3 à 5 ans avec 35 cas $(61,4 \%)$ suivie de celle entre 0 et 2 ans avec 20 cas( $35,1 \%)$, et 2 patients (3,5\%) avaient plus de 5 ans. Cinquante-six patients (98\%) provenaient de l'intérieur du pays et 54 patients $(94,7 \%)$ avaient un niveau socio-économique bas avec des parents non instruits. Un antécédent de traitement traditionnel a été retrouvé chez 45 patients (79\%), et 34 patients (59,7\%) avaient reçu un traitement mixte (traditionnel et médical). La notion de consanguinité a été notée chez 45 patients (78,6\%) et aucun antécédent familial de tumeur n'a été retrouvé. Le mode de début était une leucocorie dans $87,7 \%$ des cas (50 patients) et strabisme chez 7 patients soit $12,3 \%$ 
Tableau I : Répartition des patients selon le délai entre l'apparition des symptômes et la première consultation

\begin{tabular}{|c|c|c|}
\hline Délai d'apparition des symptômes (en mois) & Effectif & Pourcentage (\%) \\
\hline$\leq 3$ mois & 2 & 3,5 \\
\hline [4mois-6mois] & 6 & 10,5 \\
\hline [7mois-12mois] & 11 & 19,3 \\
\hline$\geq 12$ mois & 38 & 66,7 \\
\hline TOTAL & 57 & 100 \\
\hline
\end{tabular}

Plus de la moitié des patients (66,7\%) avaient consulté plus de 12 mois après l'apparition des symptômes. La répartition selon la latéralité a montré que 22 patients (38,59\%) avaient une atteinte de l'œil gauche, 21 patients (36,84\%) avaient une atteinte de l'œil droit et 14 patients $(24,56 \%)$ une atteinte bilatérale (figure 2). Tous nos patients étaient au stade $\mathrm{V}$ de la classification de Reese-Ellsworth. La tumeur était extraoculaire chez 54 patients soit 94,78\%(figure 1 et 3).

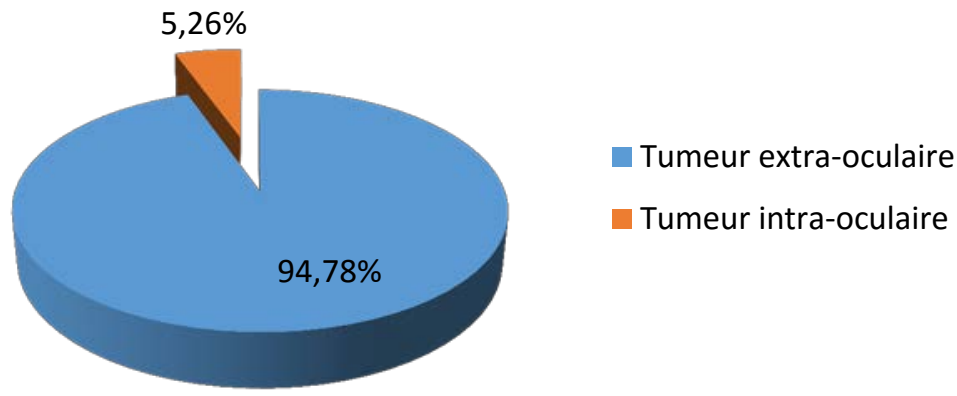

Figure 1: Repartition des patients selon l'extension tumorale.

Huit de nos patients $(14,03 \%)$ avaient eu une tomographique comme bilan d'extension. Une énucléation a été faite chez 46 patients soit 80,70\%(figure4) et 54 patients (94,73\%) avaient reçu un traitement par chimiothérapie (figure 5). L’examen anatomopathologique réalisé chez 34 patients $(59,64 \%)$ avait confirmé le diagnostic dans tous les cas.

Tableau II : Répartition selon le délai de survie après diagnostic.

\begin{tabular}{|c|c|c|}
\hline Délai survie en mois & nombre & pourcentage \\
\hline $0-6$ & 6 & 10,5 \\
\hline $7-12$ & 30 & 52,6 \\
\hline $13-18$ & 12 & 21 \\
\hline$>18$ & 9 & 15,8 \\
\hline Total & 57 & 100 \\
\hline
\end{tabular}

La survie à 7 mois était de 52,6\% et $15,78 \%$ des patients avaient survécu au-delà de 18 mois 


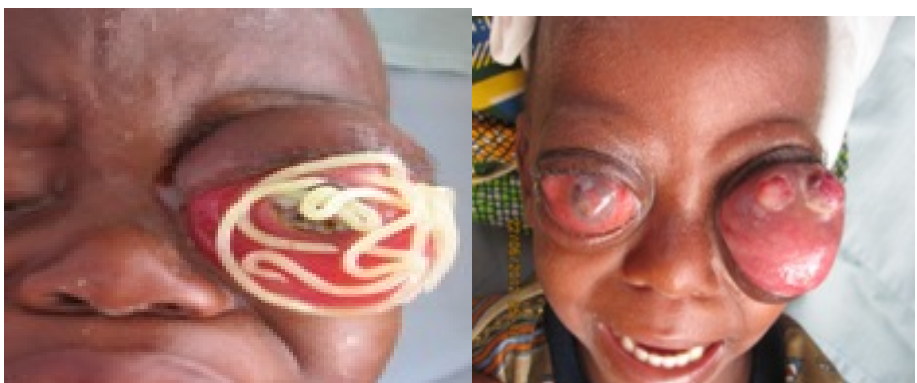

Figure 2: rétinoblastome unilateral

Figure 3: rétinoblastome bilatéral
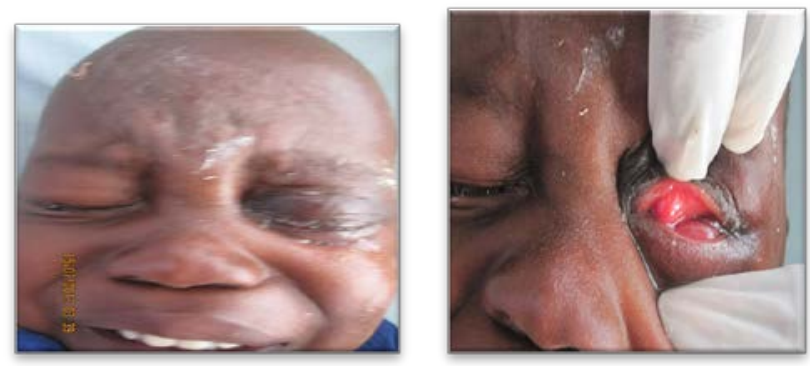

Figure 4 : patient fig. 3 après chimiothérapie

Figure 5 : même patient après énucléation

\section{Discussion et commentaires}

Le rétinoblastome survient chez les enfants porteurs de deux

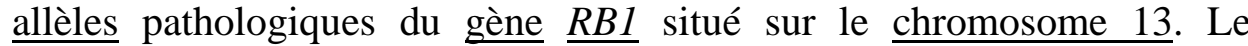
développement du cancer est dû à une mutation présente sur chacun des deux chromosomes 13 en position 13q14. La mutation peut survenir «de novo», et dans ce cas même si elle n'est pas transmise par les deux parents elle proviendrait le plus souvent d'une cellule germinale du père. Dans de rares cas, la mutation peut être de type mosaïque, c'est-à-dire, présente seulement sur certaines cellules. Les rétinoblastomes bilatéraux et multifocaux sont tous héréditaires [3]. Dans notre série 38 patients étaient de sexe masculin (sex ratio $=2$ ). Cette prédominance du sexe masculin est la règle[4-9] ; dont celle de Kazadi et al citée par Aimé et al qui ont retrouvé un sex ratio très proche du notre 2.3 [4] même si certains auteurs ont rapporté une égalité des sexes voire même une prédominance féminine. [1, 2, 10]. L'age moyen de nos patients au moment du diagnostic était de 32 mois avec des extrêmes allant de 7 mois à 6 ans. L'Age moyen des patients étaient de 42 mois dans l'étude de Kagmeni et al au Cameroun [2] et de 48 mois selon Traore et al au Mali [11]. Cependant des patients beaucoup plus jeunes âgés de 22 mois et 26,8 mois ont étés rapportés par Shridevi et al , et Bouguila et al $[8,12]$. Par contre Aziz et al aux États Unis rapportent que 79\% de leurs patients étaient diagnostiqués avant l'âge de six mois [10]. Ce constat témoigne le fait que dans les pays à faible revenu, le diagnostic du rétinoblastome est 
posé à un stade tardif. La présentation clinique du rétinoblastome est disparate. Dans notre étude comme pour d'autres auteurs [1, 2, 8, 10, 1315], la leucocorie était le signe d'appel le plus fréquent. Les autres signes d’appels peuvent être une exophtalmie (retrouvé par Aimé et al) [4] ou une baisse de l'acuité visuelle rapporté par Shuwei et al [9]. Dans notre sous-région, le recours au traitement traditionnel est la première option pour bon nombre de malades. Son accessibilité, son moindre cout et les considérations socioculturelles y contribuent fortement. Ce fait est en partie responsable du retard à la consultation qu'accusent les patients. Le traitement traditionnel avait été le premier traitement dans $79 \%$ des cas chez nos patients. Gray Kanteng et al ont rapporté que 91\% de leurs patients avaient consulté tardivement, soit parce qu’ils ont été référés en retard ou parce qu’ils ont erré auprès des praticiens de médecine traditionnelle [16]. Dans leur série Abdu et al au Nigeria ont montré que $33 \%$ des patients ont eu recours au traitement traditionnel [10].Ce aspect a été aussi abordé dans l'étude de Reddy et al et Sharen et al [1,17]. Nous avons retrouvé une notion de consanguinité entre les parents chez 78,6\% des cas dont 51\% au premier degré, et 100\% des cas étaient sporadique avec aucun antécédent familial de tumeur oculaire. La difficulté liée au diagnostic tardif du cancer de l'enfant est commune à beaucoup de pays africains, plus de $66 \%$ de nos patients ont consulté plus d'un an après l'apparition des premiers symptômes. Ceci est probablement lié au fait que la population locale fréquente mal les centres de santé et que bon nombre font le tour des guérisseurs traditionnels avant de penser à aller à l'hôpital [1, 2, 4, 10,11]. L’atteinte était unilatérale chez 75,43\% de nos patients, ceci est le cas dans beaucoup d'études [1-18]. Les cas bilatéraux représentaient 24,56\% des cas de notre série. Des chiffres plus élevés ont été rapportés aux USA (41,5\%), en Australie (41\%), 39,1\% par Reddy et al 38,5\% par Faranoush et al, 37\% en Inde [1, 13,14]. Lawan et al, Epée et al, Kagmeni et al donnèrent des taux plus bas $(5 \%, 9 \%$, 12,3\%)[10,14,2]. Le rétinoblastome bilatéral est toujours familial, le taux élevé de consanguinité chez nos patients peut être à la base du taux élevé de rétinoblastome bilatéral dans notre série. L’œil gauche est le plus atteint avec 38,59\%, ceci corrobore les conclusions de Aimé et al (40\%), Lawan et al(52\%) et Epée et al(63\%)[4,10,14]. cliniquement tous nos patients étaient au stade $\mathrm{V}$ de la classification de Reese. L'étude de la présentation clinique montre que 94,78\% avaient une tumeur extraoculaire et seulement 5,4\% avaient une tumeur intraoculaire. Cette présentation avancée du rétinoblastome est commune pour les pays en développement. Ainsi elle est de 91,8\% en RDC, 84,6\% au Nigéria, 75\% au Cameroun, 52,8\% au Pakistan et 44,2\% au Népal. Elle est seulement de $0,5 \%$ aux USA ,1,4\% en Corée et nulle en Australie et à Singapour [1, 
2, 10, 14,16-18]. Le choix du protocole thérapeutique est fonction de la présentation clinique. L'énucléation était la seule option thérapeutique disponible dans notre contexte, elle a permis une exérèse complète chez 46 patients. En cas d'envahissement des marges de résection, notamment du nerf optique et de la choroïde, une chimiothérapie adjuvante post-énucléation était recommandée. Pour les cas extraoculaires la chimiothérapie première était utilisée pour diminuer la masse tumorale et permettre l'énucléation dans un second temps. Les problèmes socioculturels liés à la perte d'un œil, la pauvreté et la désinformation des parents seraient à l'origine des longs délais entre le moment du diagnostic et l'acceptation de l'énucléation. Ces mêmes facteurs ajoutés aux décès probable de certains patients expliqueraient le taux élevé de perdu de vue(10,7\%) enregistré dans cette étude. Tous ces facteurs de gravités (le retard à la consultation, le traitement traditionnel, la consanguinité, le stade avancé des cas, l'altération de l'état général des patients) ont contribué au fort taux de mortalité chez ces jeunes patients, au décours de cette étude seuls $15 \%$ des patients étaient en vie.

\section{Conclusion}

Dans le rétinoblastome bien que les signes d'appel soient identifiables précocement par les parents et le personnel de santé, le diagnostic du rétinoblastome se fait tardivement dans notre contexte. L'éducation de la population sur l'importance de ces signes revêt un intérêt capital pour la précocité du diagnostic et de la prise en charge de cette tumeur. Tout strabisme congénital et la leucocorie doivent faire suspecter un rétinoblastome et justifier une consultation d'ophtalmologie. L'ouverture d'un registre national du rétinoblastome est indispensable pour planifier et cordonner la prise en charge des cas.

\section{References:}

Reddy S.C, Anusya S; Clinical presentation of retinoblastoma in Malaysia: a review of 64 patients. Int Jor Ophtalmol 2010; 3(1): 64-68.

Kagmeni G, Nguefack F, Monebenimp F, Kouogang G, Ngounou F et al; Le rétinoblastome dans la région de l'ouest Cameroun : aspects cliniques, histologiques et thérapeutiques; Health Sci. Dis, 2013, 6, 14 (2).

Doz F ; Rétinoblastome : aspects récents ; Archives de pédiatrie 13(2006) 1329-1337.

Aime K.L, Michel N.A, Bertin K.T, Morse M.M, Jean Lamberti Ehungu G et al Retinoblastoma in the democratic republic of Congo: 20-year review from a tertiary hospital in Kinshasa; Journal of Cancer Epidemiology, 2012. 
Kaimbo W.K, Mvitu M.M, Missotten L; Presenting signs of retinoblastoma in Congolese patients, Bulletin de la Societe Belge d’Ophtalmologie, 2002, 283, 37-41.

Bekibele C.O, Ayede A.I, Asaolu O.O, Brown B.J; Retinoblastoma: the challenges of management in Ibadan, Nigeria, Journal of Pediatric Hematology/Oncology, 2009, 31(8), 552-555.

Peko J.F, Moyen G, Gombe-Mbalawa C ; Les tumeurs solides malignes de l'enfant à Brazzaville: aspects épidémiologiques et anatomo-pathologiques, Bull Soc Pathol Exot, 2004, 97, 2, 117-118.

Shridevi S, Jamalia R, Norazah A.R, Sunder R, Nirmala B.P et al; Presentation of retinoblastoma patients in Malaysia; Asian Pacific Journal of Cancer Prevention, 2015, 15.

Shuwei B, Ruojin R, Bin Li, Xiaolin X, Bowen Z, Fei G et al; Delay in the diagnosis of retinoblastoma in China; Acta Ophthalmologica 2011: 89: e72e74.

Lawan A, Sani M; Clinicopathological pattern and management of retinoblastoma in Kano, Nigeria; Annals of African Medicine, 2011, 10(3).

Traore F, Togo B, Sylla F, Cheick TB, Diakité A.A et al: Retinoblastoma: inventory in Mali and program to develop early diagnosis, treatments and rehabilitation: Bull Cancer. 2013:100(2):165-5.

Bouguila H, Malek I, Boujemaa C, Mouelhi M, Daghfous F et al ; Le pronostic du rétinoblastome à propos de 50 cas; J Fr. Ophtalmol, 2001 :24, 10, 1053-1056.

Faranoush M, Hedayati A.A, Mehrvar A, Mehrvar N, Zangooei R; Consequences of delayed diagnosis in treatment of retinoblastoma, Iranian Journal of Pediatrics, 2014, 8, 24(4), 381-386.

Epée E, Moukouri E, Koki G, Pondy A, Mbassi K; Présentation clinique et pronostic du rétinoblastome au Centre Hospitalier et Universitaire de Yaoundé ; Health Sci. Dis: 2014, 7, 15 (3).

Sebai L, Marraghi S, Zghal I, Khiari D, Ben Thman M et al ; Le Rétinoblastome du grand enfant ; Revue Maghrébine de Pediatrie 1994, 12, 4(B).

Gray K, Wakamb A, Gayllord M.N, Robert L, Mbuli B et al; Problématique de la prise en charge du cancer de l'enfant : expérience du rétinoblastome à Lubumbashi (RD Congo) et importance du diagnostic précoce, Pan African Medical Journal. 2013; 14: 64.

Sharan M, Mazanah M; Roles traditional healers play in cancer treatment in Malaysia: implications for health promotion and education, Asian Pacific Journal of Cancer Prevention, 2013, 14, 3593-3601.

Israels T, Ribeiro R.C, Molyneux E.M; Strategies to improve care for children with cancer in Sub-Saharan Africa. Eur J Cancer. 2010, 7; 46(11):1960-6. 